\title{
Error estimates for the Ginzburg-Landau approximation
}

By Guido Schneider, Institut für Angewandte Mathematik, Universität Hannover, D-30167 Hannover, Germany

\section{Introduction}

We consider evolutionary problems over a domain with one unbounded space-direction. Unbounded domains are a good mathematical idealization for large extended physical systems in which the typical wavelength is small compared to the size of the domain. We are interested in the behavior of dissipative systems close to the threshold of instability. If a spatially homogeneous solution becomes unstable, a whole band of Fourier modes with positive growth rates appears. In classical bifurcation theory with discrete spectrum the bifurcating solutions can be described by a finite dimensional system (ODE) using center manifold theory (see e.g. [VI92]). In our case the spectrum of the linearization at the trivial state is continuous. Hence two new problems appear: First the critical eigenspace is infinite dimensional and second it can not be separated from the uncritical part by a spectral gap. Thus center manifold theory is no longer available. One way to handle such systems is given in [Mi92] leading to PDE's with nonlocal terms. Another way is the so-called Ginzburg-Landau formalism [IMD89] which is based on multiple scaling. A formally derived PDE called the Ginzburg-Landau equation takes the role of the reduced problem in the critical subspace. It is the purpose of this paper to provide exact estimates between the solutions obtained via the Ginzburg-Landau formalism and the exact solutions. This question was first treated in [CE90] for the Swift-Hohenberg equation. A simpler proof was given in [KSM92] for cases in which the nonlinearity begins with cubic terms. The case of quadratic nonlinearities is more difficult: A first result in this case was given by $A$. van Harten [vH91] for scalar problems with analytic initial data. The case of scalar equations and initial conditions in $C_{b}^{n}(\mathbb{R}, \mathbb{C})$ was handled in [Sch92b]. The present paper extends the method used there to vector-valued problems, in particular to parabolic systems on infinite cylindrical domains. Furthermore, new research ([vH92], [Eck92]) shows that the solutions described via the Ginzburg-Landau formalism are near the attractor of the system. 
To describe the situation in which such an approximation by a Ginzburg-Landau equation as modulation equation is possible we consider as an example Bénard's problem in a strip. Our method is based on an abstract approximation theorem which is proved to cover Bénard's problem.

Bénard's problem is concerned with heat conduction and convection in a fluid contained between two plates. The lower plate is heated to constant temperature $T_{0}$, the upper plate is cooled to constant temperature $T_{1}<T_{0}$. If the difference $T_{0}-T_{1}$ is smaller than a critical value the transport of heat is done by conduction. If the difference is greater than this critical value the system develops convection to transport the heat from bottom to top. The system exhibits patterns, like rolls, or hexagons and rectangles, if we look at the three-dimensional problem. The patterns are not perfectly periodic in space and in some regions of small intensity. But we expect them to be only small modulations in time and in space of the periodic pattern. Such solutions are the subject of the Ginzburg-Landau formalism based on multiple scaling.

The mathematical equations describing Bénard's problem in a strip $\mathbb{R} \times(0, \pi)$ are the Navier-Stokes equation combined with the heat equation. The coordinates are denoted by $y \in \mathbb{R}$ and $z \in(0, \pi)$. The velocity of the liquid in $y$-direction is called $v$ and in $z$-direction $w$. Furthermore we have the temperature $\theta$ and the pressure $p$. The parameters are given by $\varrho=\beta_{0}\left(T_{0}-T_{1}\right) h^{3} /\left(\pi^{3} v^{2}\right)$ and $\kappa=\delta / v$, wherein $\delta$ stands for the heat conductivity and $v$ for the viscosity. The buoyancy force depends linearly on the temperature through $c_{0}+\beta_{0} T$. Abbreviating $u=(v, w, \theta)$,

$$
\begin{aligned}
& L_{0} u=\left(\begin{array}{c}
v_{y y}+v_{z z} \\
w_{y y}+w_{z z} \\
\kappa\left(\theta_{y y}+\theta_{z z}\right)
\end{array}\right), \quad L_{1} u=\left(\begin{array}{c}
0 \\
\varrho \theta \\
w
\end{array}\right), \quad G p=\left(\begin{array}{c}
p_{y} \\
p_{z} \\
0
\end{array}\right), \\
& N(u)=\left(\begin{array}{c}
-v v_{y}-w v_{z} \\
-v w_{y}-w w_{z} \\
-v \theta_{y}-w \theta_{z}
\end{array}\right),
\end{aligned}
$$

the equations governing Bénard's problem in a strip are given in non-dimensional form as

$$
\begin{aligned}
& \left.\begin{array}{l}
\partial_{t} u=L_{0} u+L_{1} u+G p+N(u) \\
0=D u=v_{y}+w_{z}
\end{array}\right\} \text { for }(t, y, z) \in[0, \infty) \times \mathbb{R} \times(0, \pi), \\
& v_{z}=w=\theta=0 \quad \text { for }(y, z) \in \mathbb{R} \times\{0, \pi\} .
\end{aligned}
$$

Moreover, we demand vanishing mean flux

$$
[v](y)=\int_{0}^{\pi} v(y, z) d z=0
$$

for all $y \in \mathbb{R}$. 
To write (1) as a dynamical system we eliminate the pressure term $G p$. We do that by working in the space of functions $u$ with $D u=0$. A good choice for our purposes is a closed subspace of the space of uniformly locally square-integrable functions

$$
\begin{aligned}
X & =L_{l ; u}^{2}\left(\mathbb{R} \times(0, \pi), \mathbb{R}^{3}\right) \\
& =\left\{u \mid\|u\|_{X}=\sup _{t}\left(\int_{t}^{t+1} \int_{0}^{\pi}|u(y, z)|^{2} d z d y\right)^{1 / 2}<\infty\right\}
\end{aligned}
$$

which contains all $L^{\infty}$-solutions, like fronts, spatially quasiperiodic functions and many more. Note the use of the space $L_{l ; u}^{2}$ in contrast to $C_{b}^{n}(\mathbb{R}, \mathbb{C})$ which was used in [Sch92b]. This new choice is very useful for problems on multidimensional domains, it simplifies the application of our abstract theorem greatly. We establish the existence of a projection $Q$ from $X=$ $L_{l, u}^{2}\left(\mathbb{R} \times(0, \pi), \mathbb{R}^{3}\right)$ to $Q X=X \cap\left\{D u=0,\left.w\right|_{z=0, \pi}=0,[v](y)=0 \quad \forall y \in \mathbb{R}\right\}$, with $Q G p=0$. And so (1) transforms into a dynamical system

$$
\partial_{t} u=Q L u+Q N(u)
$$

in $Q X$ under the above boundary conditions, where $L=L_{0}+L_{1}$.

To motivate the appearance of nearly spatially periodic patterns, we look first at the linearized problem. It has eigenfunctions in $Q X$ of the form

$$
\begin{aligned}
& \varphi_{m}^{ \pm}(k, \varrho) e^{i k y}=\left(-i k \cos m z,-k^{2} \sin m z / m,\right. \\
& \left.-s\left(s+\lambda \frac{ \pm}{m}\right) \sin m z /(\varrho m)\right) e^{i k y}, \quad(m \in \mathbb{N}),
\end{aligned}
$$

where $s=k^{2}+m^{2}$ and the associated eigenvalues $\lambda_{m}^{ \pm}$are given by

$$
2 \lambda_{m}^{ \pm}=-(\kappa+1) s \pm \sqrt{(\kappa-1)^{2} s^{2}+4 \varrho k^{2} s^{-1}} .
$$

We want to look at the problem close to the threshold of instability. Therefore, we fix $\kappa$ and vary the control parameter $\varrho$. Because of the demand of vanishing mean flux we have $m \geq 1$. We see that the trivial solution is unstable for $\varrho=\varrho_{c}+\varepsilon^{2}>\varrho_{c}=27 \kappa / 4$, since $\lambda_{1}^{+}\left(k, \varepsilon^{2}\right)$ is positive close to $\pm k_{c}= \pm 1 / \sqrt{2}$.

Defining the small bifurcation parameter $\varepsilon$ in this way, we see that in a $\mathcal{O}(\varepsilon)$-neighborhood of wave numbers around $\pm k_{c}$ the Fourier modes increase like $\exp \left(\varepsilon^{2} t\right)$. These modes are called critical in the following. Translating this from Fourier space into the physical space $X$, we are led to the scalings $Y=\varepsilon y$ and $T=\varepsilon^{2} t$, and to the ansatz

$$
\begin{aligned}
u & =\psi_{0}(y, z, t, \varepsilon)+\mathcal{O}\left(\varepsilon^{2}\right) \\
& =\varepsilon\left(A(Y, T) \varphi_{1}^{+}\left(k_{c}, \varrho_{c}\right) e^{i k_{c} y}+c . c .\right)+\mathcal{O}\left(\varepsilon^{2}\right), \quad(t \geq 0, y \in \mathbb{R}) .
\end{aligned}
$$

Inserting this into (2) we find by a formal calculation that the complex amplitude $A$, which modulates the spatially periodic pattern $\varphi_{1}^{+}\left(k_{c}, \varrho_{c}\right) e^{i k_{c} y}$ 
slowly in time and in space, has to satisfy the Ginzburg-Landau equation

$$
\partial_{T} A=\frac{2}{9(\kappa+1)} A+\frac{4 \kappa}{\kappa+1} \partial_{Y}^{2} A-\frac{1}{8(1+\kappa)} A|A|^{2} .
$$

This approach is called the Ginzburg-Landau formalism. It was introduced by Newell and Whitehead (see [NW69]) in 1969 for Bénard's problem and in another way by di Prima, Segal and Eckhaus (see [diPES71]) in 1971.

The question arises how good is such an approximation? Clearly we have to show that the error $u-\psi_{0}$ is small compared with a real solution on a $\mathcal{O}(1)$-time scale of the Ginzburg-Landau equation (4). For the case of Bénard's problem our theorem specializes to

Theorem 1. Let $A=A(Y, T) \in C\left(\left[0, T_{0}\right], H_{l: u}^{3}(\mathbb{R}, \mathbb{C})\right)$ be a solution of the Ginzburg-Landau equation (4). Then there exists $\varepsilon_{0}, C>0$, such that for all $0<\varepsilon<\varepsilon_{0}$ solutions $u(y, z, t, \varepsilon)$ of Bénard's problem (2) exist with

$$
\sup _{t \in\left[0, \varepsilon-2 T_{0}\right]}\left\|u(y, z, t, \varepsilon)-\psi_{0}(y, z, t, \varepsilon)\right\|_{L_{i ; u}^{2}\left(\mathbb{R} \times(0, \pi), \mathbb{R}^{3}\right)}=\mathcal{O}\left(\varepsilon^{3 / 2}\right) .
$$

The space $H_{l, u}^{3}(\mathbb{R}, \mathbb{C})$ consists of all functions $A: \mathbb{R} \rightarrow \mathbb{C}$ for which the derivatives $\partial_{Y}^{j} A$ are in $L_{l, u}^{2}(\mathbb{R}, \mathbb{C})$ for $j \leq 3$. The result is an estimate on the required time-interval $\left[0, T_{0} / \varepsilon^{2}\right]$. The error or order $\mathcal{O}\left(\varepsilon^{3 / 2}\right)$ is small compared to the solution $u$ and the approximation $\psi_{0}$ which are of order $\mathcal{O}(\varepsilon)$.

The plan of the paper is as follows: In Section 2 we describe the general framework, the assumptions and the main result. The proof is given in Section 3, it follows the lines developed in [Sch92b], but takes care of the difficulty arising from the vector-valuedness of $u(y, \cdot, t)$. In Section 4 Bénard's problem is treated by checking the assumptions of Section 2 .

Throughout this paper constants are uniformly denoted by $C$. The Fourier transform of a term $A$ will be denoted by $\hat{A}$.

\section{The general situation}

The situation of the introduction can appear in more general problems. We consider partial differential equations defined over a cylindrical domain $\mathbb{R} \times \Omega$, where $\Omega$ is a bounded domain of $\mathbb{R}^{d}$. The coordinates are given by $(y, z) \in \mathbb{R} \times \Omega$. As already said in the introduction we will examine this system as a dynamical system. To include the Navier-Stokes equation, we have to introduce a projection $Q$ which allows to work in closed subspaces of a Banach space, like the divergence free vector fields. In the last section the following abstract set-up will be applied to Bénard's problem.

Let us first introduce some function spaces: 
Let $Z_{i}\left(i=0, \ldots, r_{Z}\right)$ be a sequence of Hilbert spaces, where $Z_{i+1}$ is continuously imbedded in $Z_{i}$ with $Z_{r_{Z}}$ dense in $Z_{0}$. Then we define the space of $Z_{k}$-valued locally uniformly square integrable functions

$$
L_{l ; u}^{2}\left(Z_{k}\right)=\left\{u: \mathbb{R} \rightarrow Z_{k} \mid\|u\|_{L_{l ; u}^{2}\left(Z_{k}\right)}=\sup _{t}\left(\int_{t}^{t+1}\|u(y)\|_{Z_{k}}^{2} d y\right)^{1 / 2}<\infty\right\} .
$$

The space $H_{l ; u}^{i}\left(Z_{k}\right)$ consists of all functions $u: \mathbb{R} \rightarrow Z_{k}$ for which the derivatives $\partial_{y}^{j} u$ are in $L_{l ; u}^{2}\left(Z_{k}\right)$ for $j \leq i$. We consider now

$$
X=L_{l, u}^{2}\left(Z_{0}\right) \text { and } X^{1}=\bigcap_{j=0, \ldots, r_{Z}} H_{l ; u}^{j}\left(Z_{r_{Z}-j}\right) .
$$

All spaces are equipped with the natural norm. Since we use Fourier transform we do not distinguish between a space and its complex extension.

We assume now the following situation:

Let $L \in \mathscr{L}\left(X^{1}, X\right)$ be a linear differential operator. This operator depends smoothly on a small parameter $\varepsilon^{2}$. Next we have a Banach space $Y$ with $X^{1} \subset Y \subset X$ continuously and a nonlinear mapping $N \in C^{4}(Y, X)$ which is local in the $y$-variable. The quadratic and cubic terms of the nonlinearity can be written as

$$
\sum_{j=1}^{\text {finite }} B_{j} u \cdot C_{j} u \text { resp. } \sum_{j=1}^{\text {finite }} D_{j} u \cdot E_{j} u \cdot F_{j} u,
$$

where $B_{j}, \ldots, F_{j}$ are linear differential operators and $\cdot$ denotes the componentwise multiplication. All considered operators do not depend explicitly on $y$. To include Navier-Stokes equation we assume

(Al) There exists a family of projections $\hat{Q} \in C_{b}^{2}\left(\mathbb{R}, \mathscr{L}\left(Z_{0}, Z_{0}\right)\right)$.

We show later that we can define a projection $Q \in \mathscr{L}(X, X)$ by this family $\mathbb{R} \rightarrow \mathscr{L}\left(Z_{0}, Z_{0}\right)$. It is our aim to show a general version of Theorem 1 for systems

$$
\partial_{t} u=Q L\left(\varepsilon^{2}\right) u+Q N\left(\varepsilon^{2}, u\right)
$$

in the Banach space $Q X=\{u \in X \mid Q u=u\}$. For notational simplicity we suppress the explicit dependence of $N$ on $\varepsilon^{2}$. Moreover, we define the linear operator $\Lambda=Q L$. We assume now

(A2) $\Lambda$ is a closed unbounded operator on $Q X$ with domain of definition $D(\Lambda)=(Q X)^{1}$. Moreover, there exists $0 \leq \beta<1$, such that $(Q X)^{\beta} \subset Y$, where the space $(Q X)^{\beta}$ is an interpolation space between $Q X$ and $(Q X)^{1}$ which is equipped with the graphnorm of $\Lambda^{\beta}$. 
The main point in applying the Ginzburg-Landau approximation is the form of the spectrum. Since the problem does not explicitly depend on $y$, the eigenfunctions have the form $\hat{\varphi}_{k} e^{i k y}$ with $\hat{\varphi}_{k} \in Z_{0}$. Since $u \in L_{l ; u}^{2}$ is a tempered distribution it is possible to define its Fourier transform. Therefore, we define for $\hat{u} \in Z_{r_{Z}}$ the operator $\hat{\Lambda}\left(k, \varepsilon^{2}\right)(\hat{u})=e^{-i k y} \Lambda\left(e^{i k y} \hat{u}\right)$. The spectrum of $\hat{\Lambda}\left(k, \varepsilon^{2}\right): Z_{r_{Z}} \cap\{\hat{Q}(k) \hat{u}=\hat{u}\} \rightarrow Z_{0} \cap\{\hat{Q}(k) \hat{u}=\hat{u}\}$ is denoted by $\Sigma\left(k, \varepsilon^{2}\right)$. It is assumed for $k>0$.

(A3) There exists $k_{c}, \varrho, \varepsilon_{0}>0$, such that for $0<\varepsilon<\varepsilon_{0}$ and $\left|k-k_{c}\right|<\varrho<$ $k_{c} / 3$ the spectrum splits into two parts $\Sigma\left(k, \varepsilon^{2}\right)=\Sigma^{-}\left(k, \varepsilon^{2}\right) \cup\left\{\tilde{\lambda}\left(k, \varepsilon^{2}\right)\right\}$. Related to the curve $\tilde{\lambda}\left(\cdot, \varepsilon^{2}\right) \in C^{\infty}\left(\left(k_{c}-\varrho, k_{c}+\varrho\right), \mathbb{C}\right)$ of single eigenvalues exists a curve of eigenfunctions $\tilde{\varphi}\left(\cdot, \varepsilon^{2}\right)$. Around $k_{c}$ this curve has an expansion

$$
\tilde{\lambda}\left(k_{c}+\varepsilon K, \varepsilon^{2}\right)=i \omega_{0}+\varepsilon i v_{1} K+\varepsilon^{2}\left(\lambda_{0}+i v_{0}\right)+\varepsilon^{2} K^{2}\left(\lambda_{2}+i v_{2}\right)+\mathcal{O}\left(\varepsilon^{3}\right)
$$

uniformly for $K \in[-1,1]$ with $\lambda_{0}>0$ and $\lambda_{2}<0$. Moreover, there exists an $\varepsilon$-independent constant $\sigma>0$ such that for $0<\varepsilon<\varepsilon_{0}$

$$
\sup \Re\left(\bigcup_{k} \Sigma\left(k, \varepsilon^{2}\right) \backslash\left\{\underset{k:\left|k-k_{c}\right|<e}{ } \tilde{\lambda}\left(k, \varepsilon^{2}\right)\right\}\right)<-\sigma .
$$

Since we deal with a real problem we have $\tilde{\lambda}(k)=\overline{\bar{\lambda}}(-k)$ resp. $\tilde{\varphi}(k)=\overline{\tilde{\varphi}}(-k)$. To solve (5) at least locally in time it is natural to assume that $\Lambda$ is the generator of an analytic semigroup in $Q X$. We additionally need exact estimates for the exponential decay rates of the semigroup applied to different Fourier modes.

(A4) There exists $a \in \mathbb{R}, n \in \mathbb{N}, \delta_{0}, C_{1}, C_{2}>0$, such that we have for $s \in$ $S(k)=\left\{s \in \mathbb{C} \mid \arg \left(s+a+\left(C_{1} / 2\right) k^{2 n}\right) \in\left(-(\pi / 2)-\delta_{0},(\pi / 2)+\delta_{0}\right)\right\}$ and $0<$ $\varepsilon<\varepsilon_{0}$ the estimates

(a) $\left\|(\hat{\Lambda}(k)-s)^{-1}\right\|_{\mathscr{L}\left(Z_{0}, Z_{0}\right)}<C_{2} / \mid s+a+1+C_{1} k^{2 n}$,

(b) $\sup _{k \in \mathbb{R}, s \in S(k)}\left\|\hat{\Lambda}^{\prime}(k)(\hat{\Lambda}(k)-s)^{-1}\right\|_{\mathscr{L}\left(Z_{0}, Z_{0}\right)}<C$,

(c) $\sup _{k \in \mathbb{R}, s \in S(k)}\left\|\hat{\Lambda}^{\prime \prime}(k)(\hat{\Lambda}(k)-s)^{-1}\right\|_{\mathscr{L}\left(Z_{0}, Z_{0}\right)}<C$.

Contrary to the introduction the imaginary coefficients $\omega_{0}$ and $v_{1}$ appearing in assumption (A3) give rise to fast dynamics. We get rid of $v_{1}$ by the transformation $x \mapsto x-v_{1} t$. The coefficient $\omega_{0}$ is taken into account in the approximation

$$
\psi_{0}(y, z, t, \varepsilon)=\varepsilon\left(A_{1}(Y, T) \tilde{\varphi}\left(k_{c}, 0\right) e^{i k_{c} y+i \hat{\omega}_{0} t}+c . c .\right),
$$

where $\omega_{0}-v_{1} k_{c}=: \hat{\omega}_{0}$. The Ginzburg-Landau equation for (5) has the form

$$
\partial_{T} A_{1}=\left(\lambda_{0}+i v_{0}\right) A_{1}-\left(\lambda_{2}+i v_{2}\right) \partial_{Y}^{2} A_{1}+\gamma A_{1}\left|A_{1}\right|^{2},
$$


where $\gamma$ can be formally derived from (5) and is given for $\hat{\omega}_{0}=0$ in a later section by (9). Suppose the above situation, we can state the following theorem.

Theorem 2. Let $A_{1}=A_{1}(Y, T) \in C\left(\left[0, T_{0}\right], H_{l ; u}^{3}(\mathbb{R}, \mathbb{C})\right)$ be a solution of the Ginzburg-Landau equation (7). Then there exists $\varepsilon_{0}, C>0$, such that for all $0<\varepsilon<\varepsilon_{0}$ solutions $u(y, z, t, \varepsilon)$ of problem (5) exist with

$$
\sup _{t \in\left[0, \varepsilon-2 T_{0}\right]}\left\|u(y, z, t, \varepsilon)-\psi_{0}(y, z, t, \varepsilon)\right\|_{X}=\mathcal{O}\left(\varepsilon^{3 / 2}\right) .
$$

Due to the form of the spectrum Ginzburg-Landau approximation is for example possible in hydrodynamic problems, like Bénard's problem, Taylor-Couette problem, or Poiseuille flow, or in reaction-diffusion systems.

\section{The proof}

We start the proof with a short summary of the main ideas and steps. We write a solution $u$ of (5) as a sum of an approximation $\psi$ and an error $\varepsilon^{3 / 2} R: u=\psi+\varepsilon^{3 / 2} R$. Note the scaling of the error by $\varepsilon^{3 / 2}$ contrary to $\varepsilon^{2}$ in [Sch92b]. This is due to the choice of the norm (see also [KSM92]). Inserting this into (5) we get a differential equation for $R$ which has to be solved on the time interval $\left[0, T_{0} / \varepsilon^{2}\right]$. It has to be shown that $R$ is of order $O(1)$ on this time interval.

Former work ([vH91], [KSM92]) shows that quadratic terms in the nonlinearity are the main difficulty in establishing the stated result. The quadratic terms give rise to low $\varepsilon$-orders in front of the linear and nonlinear terms in the differential equations for the error. Whence it is more difficult to establish the estimate on the required time interval $\left[0, T_{0} / \varepsilon^{2}\right]$.

This difficulty can be overcome by separating linearly exponentially damped modes from linearly unstable, called critical, modes and using different scalings for them. This method is presented in [Sch92b] and we refer to this paper for an introduction. The key observation is that the convolution of critical Fourier modes (wave numbers concentrated around $\pm k_{c}$ ) gives Fourier modes with wave numbers concentrated around $0, \pm 2 k_{c}$ (see Lemma 9) which are damped. Thus, the previously dangerous terms (with low $\varepsilon$-order) only appear in the damped part where they can be controlled easily (see also system (15)).

One basic tool is Fourier transform with respect to the unbounded direction. As discussed above we work in $L_{l ; u}^{2}$, since this space is close to $L^{2}$ (where Fourier transform is an isomorphism), but also contains $L^{\infty}$. We pull back linear problems from $L_{l ; \mu}^{2}$ to $L^{2}$ by localization and use in applications already known facts about the system which has to be handled. As another basic tool we use abstract semigroup theory via the Dunford 
integral. Assumption (A4), which is trivially fulfilled for scalar equations, is needed to construct the analytic semigroup on $L_{l ; u}^{2}$.

The approximation $\psi_{0}$ given in (6) is not contained completely in the critical subspace. For the estimation of the error we will replace $\psi_{0}$ by another approximation $\psi$ which lies completely in the critical subspace and satisfies $\left\|\psi_{0}-\psi\right\|_{L_{l ;}^{2}\left(Z_{0}\right)}=\mathcal{O}\left(\varepsilon^{2}\right)$. In the vector-valued case a new difficulty appears here, since the eigenfunctions vary with the wave number $k$.

We start the proof with an explanation how we use Fourier transform in $L_{l ; u}^{2}$. Next we construct the mode filters to separate the critical modes. After that we construct an approximation $\psi$ which lies in $Q X$ and has all desired properties. Then we estimate the error with a method similar to [Sch92b], except that some new technical details appear.

\subsection{The Fourier transform set-up}

Fourier transforming a function $u \in L_{l ; u}^{2}$ gives a tempered distribution, for which it is not so easy to define actions of operators in Fourier space and to estimate later on their norms in the physical space. To handle this problem, we use functions in weighted spaces.

Definition 3. Let $W$ be a Banach space and $H^{m}(W)$ the Sobolev space $H^{m}(\mathbb{R}, W)$. We define the space $H^{m}(W, n)(m, n \in \mathbb{N})$ by

$$
u \in H^{m}(W, n) \Leftrightarrow u(\cdot)\left(1+|\cdot|^{2}\right)^{n / 2} \in H^{m}(W) .
$$

This space is equipped with the natural norm.

Clearly, we write $L^{2}$ for $H^{0}$. We will make use of the fact that Fourier transform

$$
\hat{u}(k)=(\mathscr{F} u)(k)=\frac{1}{2 \pi} \int e^{-i k y} u(y) d y
$$

is an isomorphism between $H^{m}(W, n)$ and $H^{n}(W, m)$, if $W$ is a Hilbert space and $m, n \in \mathbb{N}_{0}$. We see that the decay of the functions in Fourier space is related to the smoothness in physical space and vice versa.

Our method depends heavily on Fourier transform with respect to the axial variable $y$. The great advantage appears in the fact that the linear part is a local operator in the wave number $k$. We call operators multipliers if they are defined by multiplying $\hat{u}=\mathscr{F} u$ by a family of linear operators $\hat{M} \in L^{\infty}\left(\mathbb{R}, \mathscr{L}\left(W_{1}, W_{2}\right)\right)$ with $W_{1}, W_{2}$ Hilbert spaces, i.e.

$$
\hat{M}: L^{2}\left(W_{1}\right) \rightarrow L^{2}\left(W_{2}\right) ; \hat{u} \mapsto \hat{M}(\cdot) \hat{u}(\cdot) .
$$


The associated operator in the physical space is denoted by

$$
M: L^{2}\left(W_{1}\right) \rightarrow L^{2}\left(W_{2}\right) ; u \mapsto \mathscr{F}^{-1}(\hat{M} \mathscr{F} u) .
$$

Obviously $M$ is translation invariant, i.e. $\tau_{h} M=M \tau_{h}$, where $\tau_{h} u(y)=$ $u(y+h)$ for all $h \in \mathbb{R}$. We have the following classical result of multiplier theory.

Lemma 4. Let $m \in Z$ and $k \mapsto\left(1+k^{2}\right)^{m / 2} \hat{M}(k) \in C_{b}^{n}\left(\mathbb{R}, \mathscr{L}\left(W_{1}, W_{2}\right)\right)$, where $W_{1}, W_{2}$ are Hilbert spaces. Then $M: H^{q}\left(W_{1}, n\right) \rightarrow H^{q+m}\left(W_{2}, n\right)$ is bounded for all $q \in \mathbb{N}_{0}$ with $q+m \geq 0$ and norm $\leq C(q, n, m) \|(1+$ $\left.|\cdot|{ }^{2}\right)^{m / 2} \hat{M} \|_{C_{b}^{n}\left(\mathbb{R}, \mathscr{L}\left(W_{1}, W_{2}\right)\right)}$, where $C(q, n, m)$ does not depend on $\hat{M}$.

The proof follows immediately from the observation that $\hat{M} \epsilon$ $\mathscr{L}\left(H^{n}\left(W_{1}, q\right), H^{n}\left(W_{2}, q+m\right)\right)$ and from the isomorphism property of Fourier transform.

We are interested in similar results for operators on $H_{l ; u}^{q}\left(W_{j}\right)$. When does a multiplier $\hat{M}(k) \in \mathscr{L}\left(W_{1}, W_{2}\right)$ define a bounded linear operator $M_{i ; u}$ from $H_{l ; u}^{q}$ into $H_{l ; u}^{q+m}$ ? Of course $M_{l ; u}$ has to satisfy $\left(\mathscr{F} M_{l ; u} u\right)[\varphi]=$ $(\hat{M}(\cdot)(\mathscr{F} u))[\varphi]$ for all test functions $\varphi \in H^{2}\left(W_{2}, \max (0,-m)\right)$.

Lemma 5. Let $m \in Z$ and $k \rightarrow\left(1+k^{2}\right)^{m / 2} \hat{M}(k) \in C_{b}^{2}\left(\mathbb{R}, \mathscr{L}\left(W_{1}, W_{2}\right)\right)$. Then $M_{l ; u}: H_{l ; u}^{q}\left(W_{1}\right) \rightarrow H_{l ; u}^{q+m}\left(W_{2}\right)$ is bounded for all $q \in \mathbb{N}_{0}$ with $q+m \geq 0$ and norm

$$
\leq C(q, m)\left\|\left(1+|\cdot|^{2}\right)^{m / 2} \hat{M}\right\|_{C_{b}^{2}\left(\mathbb{B}, \mathscr{L}\left(W_{1}, W_{2}\right)\right)},
$$

where $C(q, m)$ does not depend on $\hat{M}$.

Proof. We choose $\chi \in C_{0}^{\infty}$ such that its support is contained in $[-1,1]$ and $\sum_{j \in Z} \chi(y+j) \equiv 1$. We show that $M_{l ; u}$ can be defined as follows. For $u \in H_{i ; u}^{q}\left(W_{1}\right)$ we set $u_{j}(y)=u(y-j) \chi(y)$. Since $u_{j} \in H^{q}\left(W_{1}, 2\right)$ we find $v_{j}=M u_{j} \in H^{q+m}\left(W_{2}, 2\right)$. Now let $M_{i ; u} u=\sum_{j \in Z} \tau_{j} v_{j}$. Clearly this sum does not converge in $H_{l ; u}^{q+m}\left(W_{2}\right)$. But, since $\tau_{j} v_{j}$ is concentrated around $y=j$ and decays like $1 /\left(1+(y-j)^{2}\right)$ it is easy to see that $\sum_{j \in Z} \tau_{j} v_{j}$ converges locally to a function in $H_{l ; u}^{q+m}\left(W_{2}\right)$ with norm $\leq C(q, m) \|(1+$ $\left.|\cdot|^{2}\right)^{m / 2} \hat{M}\left\|_{C_{b}^{2}\left(\mathbb{R}, \mathscr{L}\left(W_{1}, W_{2}\right)\right)}\right\| u \|_{H_{l ;}^{q}\left(W_{1}\right)}$. Therefore, we will write $l-\sum$ instead of $\sum$ to denote that this sum does not converge absolutely, but locally in $H^{q+m}\left(I, W_{2}\right)$ on every compact interval $I$.

It remains to be shown that the extension of $M$ to $M_{l: u}$ is also a multiplier. Therefore, we take a testfunction $\varphi \in H^{2}\left(W_{2}, \max (0,-m)\right)$. 
Note that $\mathscr{F} \varphi \in H^{\max (0,-m)}\left(W_{2}, 2\right)$. So we can use Fubini twice to get

$$
\begin{aligned}
\left(\mathscr{F} M_{l ; u} u\right)[\varphi] & =\left(M_{l ; u} u\right)[\mathscr{F} * \varphi] \\
& =\int\left(l-\sum_{j \in Z} \tau_{j} M u_{j}, \mathscr{F} * \varphi\right)_{W_{2}} d k \\
& =\sum_{j \in Z} \int\left(\tau_{j} M u_{j}, \mathscr{F} * \varphi\right)_{W_{2}} d k \\
& =\sum_{j \in Z} \int\left(\tau_{j} u_{j}, M^{*} \mathscr{F}^{*} \varphi\right)_{W_{1}} d k \\
& =\sum_{j \in Z} \int\left(\tau_{j} u_{j}, \mathscr{F} * \hat{M}^{*} \varphi\right)_{W_{1}} d k \\
& =\int\left(u, \mathscr{F} * \hat{M}^{*} \varphi\right)_{W_{1}} d k=(\hat{M}(\cdot)(\mathscr{F} u))[\varphi],
\end{aligned}
$$

where $M^{*}$ resp. $\mathscr{F}^{*}$ denotes the adjoint operator of $M$ resp. $\mathscr{F}$. All integrals exist, since

$$
\begin{aligned}
\sum_{j \in Z} \int\left(\tau_{j} u_{j}, \mathscr{F}^{*} \hat{M}^{*} \varphi\right)_{W_{1}} d k \leq & C \sum_{j \in Z} \sup _{y}\left|\frac{1}{(1+|y|)^{2}} \frac{1}{(1+|y+j|)^{2}}\right| \\
& \times\|u\|_{L_{l ; w^{2}}^{2}\left(W_{1}\right)}\left\|\mathscr{F} * \hat{M}^{*} \varphi\right\|_{L^{2}\left(W_{1}, 2\right)}<\infty .
\end{aligned}
$$

For simplicity we write in the following $M$ instead of $M_{i ; u}$.

\subsection{The mode filters}

To separate now for fixed wave number $k$ the critical from the uncritical eigenfunctions, we define the $\hat{\Lambda}\left(k, \varepsilon^{2}\right)$-invariant projection to the critical eigenspace. For $\hat{u} \in Z_{0}$ we do that by

$$
\tilde{E}_{c}\left(k, \varepsilon^{2}\right) \hat{u}:=\frac{1}{2 \pi i} \int_{\Gamma}\left(\hat{\Lambda}\left(k, \varepsilon^{2}\right)-\mu I d .\right)^{-1} \hat{u} d \mu,
$$

where $\Gamma$ is a closed curve around the single eigenvalue $\lambda\left(k_{c}, 0\right)=i \hat{\omega}_{0}$. By assumption (A3) this is possible for $k \in\left[k_{c}-\varrho, k_{c}+\varrho\right]$ and $0 \leq \varepsilon \leq \varepsilon_{0}$. For $k \in\left[-k_{c}-\varrho,-k_{c}+\varrho\right]$ we set $\widetilde{E}_{c}\left(k, \varepsilon^{2}\right)=\tilde{\bar{E}}_{c}\left(-k, \varepsilon^{2}\right)$. It is well known that the resolvent is analytic with respect to $\mu$ in the resolvent set of $\hat{\Lambda}(k)$. Since $\hat{\Lambda}$ is a smooth function of $k$ and $\varepsilon^{2}$ with values in $\mathscr{L}\left(Z_{r_{Z}}, Z_{0}\right)$ the resolvent is smooth as well. Thus, $\tilde{E}_{c}$ is smooth in $k$ and $\varepsilon^{2}$. Note that $\tilde{E}_{c}\left(k, \varepsilon^{2}\right)$ is a projection with one dimensional range span $\left\{\tilde{\varphi}\left(k, \varepsilon^{2}\right)\right\}$. Therefore we define for $k \in\left[-k_{c}-\varrho,-k_{c}+\varrho\right] \cup\left[k_{c}-\varrho, k_{c}+\varrho\right]$ and $\hat{u} \in Z_{0}$ a complex valued operator $\tilde{p}_{\varphi}=\tilde{p}_{\varphi}\left(k, \varepsilon^{2}\right)$ by

$$
\tilde{E}_{c}\left(k, \varepsilon^{2}\right) \hat{Q}(k) \hat{u}=\tilde{p}_{\varphi}\left(k, \varepsilon^{2}\right)(\hat{u}) \tilde{\varphi}_{ \pm 1}\left(k, \varepsilon^{2}\right) .
$$

To extend $\tilde{E}_{c}$ to a function for all $k \in \mathbb{R}$ we use an even cut-off function 
$\hat{\chi}_{0} \in C_{0}^{\infty}$ in Fourier space which is defined by

$$
\hat{\chi}_{0}(k)= \begin{cases}1 & \text { for } k \in[-\varrho / 6,+\varrho / 6], \\ 0 & \text { for } k \notin[-\varrho / 3,+\varrho / 3], \\ \in[0,1] & \text { other cases. }\end{cases}
$$

With help of this cut-off function we define now multipliers in Fourier space.

Definition. For ||$k\left|-k_{c}\right|<\varrho$ we define the multipliers

$$
\begin{aligned}
& \hat{\varphi}_{ \pm 1}\left(k, \varepsilon^{2}\right)=\tilde{\varphi}\left(k, \varepsilon^{2}\right) \hat{\chi}_{0}\left(k \mp k_{c}\right), \quad \hat{\lambda}_{ \pm 1}\left(k, \varepsilon^{2}\right)=\tilde{\lambda}\left(k, \varepsilon^{2}\right) \hat{\chi}_{0}\left(k \mp k_{c}\right), \\
& \hat{p}_{\varphi \pm 1}\left(k, \varepsilon^{2}\right)=\tilde{p}_{\varphi}\left(k, \varepsilon^{2}\right) \hat{\chi}_{0}\left(k \mp k_{c}\right) .
\end{aligned}
$$

These operators are extended by zero on whole real axis. We define the operator which extracts the critical modes by $\hat{E}_{c}=\sum_{i= \pm 1} \hat{\varphi}_{i} \hat{p}_{\varphi_{i}}$. Its counterpart to the uncritical modes is denoted by $\hat{E}_{s}=1-\hat{E}_{c}$. We call $\hat{E}_{c}$ and $\hat{E}_{s}$ filters, since they separate critical from uncritical wave numbers. The operator $E_{c}$ is no projection and so we define auxiliary mode filters

$$
\hat{E}_{c}^{h}\left(k, \varepsilon^{2}\right)=\tilde{E}_{c}\left(k, \varepsilon^{2}\right)\left(\hat{\chi}_{0}\left(k_{c}+k / 2\right)+\hat{\chi}_{0}\left(-k_{c}+k / 2\right)\right)
$$

and

$$
\hat{E}_{s}^{h}\left(k, \varepsilon^{2}\right)=\widetilde{E}_{s}\left(k, \varepsilon^{2}\right)\left(1-\hat{\chi}_{0}\left(k_{c}+2 k\right)-\hat{\chi}_{0}\left(-k_{c}+2 k\right)\right),
$$

such that $\hat{E}_{c}^{h} \hat{E}_{c}=\hat{E}_{c}$ and $\hat{E}_{s}^{h} \hat{E}_{s}=\hat{E}_{s}$. With help of Lemma 5 we can now define operators in the physical space which are denoted with the same symbol, but without $\hat{\imath}$

\subsection{Multipliers acting on scaled functions}

Next we have to estimate multipliers acting on scaled functions, such as in (6). We introduce a scaling operator $S_{\varepsilon}$ by $\left(S_{\varepsilon} u\right)(y):=u(\varepsilon y)$. One difficulty with the $L_{l ; u}^{2}$-norm is that there is no exact relation between $\left\|S_{\varepsilon} u\right\|_{L_{l ; u}^{2}}$ and $\|u\|_{L_{i: u}^{2}}$. This is due to the fact that locally this norm behaves like the $L^{2}$-norm and globally like the $L^{\infty}$-norm. Looking at $|x|^{-\alpha}$ with $0 \leq \alpha<\frac{1}{2}$ we find $\left\|S_{\varepsilon} u\right\|_{L_{l ; u}^{2}}=C \varepsilon^{-\alpha}\|u\|_{L_{l ; u}^{2}}$ for every $0 \leq \alpha<\frac{1}{2}$. So, in general we have $\left\|S_{\varepsilon} u\right\|_{L_{l ; u}^{2}} \leq C \varepsilon^{-1 / 2}\|u\|_{L_{l ; u}^{2}}^{2}$, but $\left\|S_{\varepsilon} u\right\|_{L_{l ; u}^{2}} \leq C\|u\|_{L^{\infty}} \leq C\|u\|_{H_{l ; u}^{1}}$.

Lemma 6. Let $m \in \mathbb{N}$ and $\left(1+|\cdot|^{2}\right)^{-m / 2} \hat{M}(\cdot) \in C_{b}^{2}\left(\mathbb{R}, \mathscr{L}\left(W_{1}, W_{2}\right)\right)$. Then $M\left[\left(S_{\varepsilon} \cdot\right) e^{i k_{0} y}\right]: H_{l ; u}^{q}\left(W_{1}\right) \rightarrow H_{l ; u}^{q-r}\left(W_{2}\right)$ is bounded for all $q \geq r \geq m$ with norm

$$
\begin{aligned}
& \leq C(q, m, r)\left\|\left(1+|\cdot|^{2}\right)^{-m / 2} \hat{M}\left(\varepsilon \cdot+k_{0}\right)\right\|_{C_{b}^{2}\left(\mathbb{R}, \mathscr{L}\left(W_{1}, W_{2}\right)\right)} \\
& \times\left\|S_{\varepsilon}\right\|_{\mathscr{L}\left(H_{l ; \mu}^{q-m}\left(W_{2}\right), H_{l: u}^{q-r_{(}}\right.}^{\left.\left.q-W_{2}\right)\right)},
\end{aligned}
$$

where $C(q, m, r)$ does not depend on $\hat{M}$. 
Proof. With help of test functions we get

$$
\begin{aligned}
& M\left[\left(S_{\varepsilon} u\right) e^{i k_{0} y}\right]=\mathscr{F}^{-1} \hat{M} \mathscr{F}\left[\left(S_{\varepsilon} u\right) e^{i k_{0} y}\right] \\
& =\varepsilon^{-1 \mathscr{F}-1} \hat{M}\left[\left(S_{1 / \varepsilon} \tau_{-k_{0}} \hat{u}\right)\right] \\
& =\varepsilon^{-1 \mathscr{F}-1}\left(S_{1 / \varepsilon} \tau_{-k_{0}}\left[\left(S_{\varepsilon} \tau_{k_{0}} \hat{M}\right) \hat{u}\right]\right. \\
& =S_{\varepsilon} \mathscr{F}^{-1} \tau_{-k_{0}}\left[\left(S_{\varepsilon} \tau_{k_{0}} \hat{M}\right) \mathscr{F} u\right]
\end{aligned}
$$

with new multiplier $\hat{M}_{\varepsilon}=S_{\varepsilon} \tau_{k_{0}} \hat{M}$ in Fourier space. With help of the last lemma the assertion follows.

To motivate the next lemma we look at $\partial_{y} u(\varepsilon y)=\varepsilon \partial_{Y} u(Y)=\mathscr{O}(\varepsilon)$ with $Y=\varepsilon y$. This means, differentiating scaled functions wins one order of $\varepsilon$. In this way, we compute the Ginzburg-Landau equation. To generalize this, to e.g. $\widehat{Q}(k)$, and to estimate later on the residuum (8) we show

Lemma 7. Assume the same situation as in Lemma 6 and $\hat{M}\left(k+k_{0}\right)=$ $\mathcal{O}\left(k^{s}\right)$ for $k \rightarrow 0$ and $s \leq m$. Then

$$
\left\|\left(1+|\cdot|^{2}\right)^{-m / 2} \hat{M}\left(\varepsilon \cdot+k_{0}\right)\right\|_{C_{b}^{2}\left(\mathbb{R}, \mathscr{L}\left(W_{1}, W_{2}\right)\right)}=\mathcal{O}\left(\varepsilon^{s}\right) .
$$

The proof follows easily by simple calculations. We remark that it is possible to show a similar Lemma without loss of regularity (see [Sch92a], [Sch92b]) by considering the related Green's function or by introducing spaces with scaled norms. But, for our purposes this lemma is sufficient.

\subsection{The approximation and the residuum}

Now we have defined the operators to separate the critical from the uncritical modes. So we can split the error $R$ in two parts, into a critical part $R_{c}=E_{c} R$ and an uncritical part $R_{s}=E_{s} R$ and scale it differently by $\delta_{c}(\varepsilon)$ and $\delta_{s}(\varepsilon)$.

If we insert $u=\delta_{c}(\varepsilon) R_{c}+\delta_{s}(\varepsilon) R_{s}+\psi_{0}$ into (5) new problems occur. For hydrodynamic problems $\psi_{0}$ would be in general not in $Q X$. If $\psi_{0}$ is in $Q X$, nevertheless the residuum

$$
\operatorname{Res}\left(\psi_{0}\right)=-\partial_{t} \psi_{0}+\Lambda \psi_{0}+Q N\left(\psi_{0}\right)
$$

remains in the equations for the error. Some terms of it drop out, since $A_{1}$ which appears in the approximation $\psi_{0}$ is the solution of the GinzburgLandau equation. But what remains is of too low $\varepsilon$-order to estimate the error in the required way. Therefore we have to construct an approximation $\psi, \mathcal{O}\left(\varepsilon^{2}\right)$-close to $\psi_{0}$, such that $\operatorname{Res}(\psi)$ is of order $\mathcal{O}\left(\varepsilon^{n}\right)$ for sufficiently large $n \in \mathbb{N}$. Moreover, we construct $\psi$ in such a way that we need only low regularity initial conditions for the Ginzburg-Landau equation. 
The linear part of the Ginzburg-Landau equation is determined by $\hat{\lambda}_{1}$. The coefficient $\gamma$ in front of the cubic term is only determined by the quadratic and cubic terms of the nonlinearity. Higher order terms would give rise to high $\varepsilon$-orders. So we consider

$$
\partial_{t} u=\Lambda u+B(u, u)+T(u, u, u)+\mathcal{O}\left(\|u\|^{4}\right),
$$

where $B$ is a symmetric bilinear form and $T$ a symmetric trilinear form.

The construction of the approximation is as follows. The terms of order $\mathcal{O}(\varepsilon)$ should be close to $\psi_{0}$ and in the eigenspaces, depending on $k$, to the critical modes. Therefore, we choose the approximation $\psi_{c}$ with

$$
\begin{aligned}
\psi_{c}(y, z, t, \varepsilon)= & \varepsilon \varphi_{1}\left(A_{1}\left(\varepsilon y, \varepsilon^{2} t\right) e^{i k_{c} y+i \hat{\omega}_{0} t}\right) \\
& +\varepsilon \varphi_{-1}\left(A_{-1}\left(\varepsilon y, \varepsilon^{2} t\right) e^{-i k_{c} y-i \hat{\omega}_{0} t}\right),
\end{aligned}
$$

where $A_{1}$ is the solution of the related Ginzburg-Landau equation and $\bar{A}_{-1}(Y)=A_{1}(Y)$. Note that $\varphi_{ \pm 1}$ are operators defined above via multipliers and not fixed eigenfunctions. For notational reason we assume now that $\hat{\omega}_{0}=0$. For the general case, $\hat{\omega}_{0} \neq 0$, everything that follows will work in the same way. It is only more complicated to write it down due to the appearance time derivatives of $e^{i \hat{\omega}_{0} t}$ (see also [Sch92a]).

Inserting $\psi_{c}$ in the system, $\operatorname{Res}\left(\psi_{c}\right)$ contains terms of too low $\varepsilon$-order. To eliminate these terms we extend our approximation by $\varepsilon^{2} \psi_{s}$. The term $\varepsilon^{2} \psi_{s}$ is not necessary for the approximation property, since it is of order $\mathcal{O}\left(\varepsilon^{2}\right)$, but it is helpful in making the residuum small. Inserting the approximation $\psi=\varepsilon \psi_{c}+\varepsilon^{2} \psi_{s}$ into the equation gives the residuum

$$
\begin{aligned}
\operatorname{Res}(\psi)= & -\partial_{t} \psi+\Lambda \psi+B(\psi, \psi)+T(\psi, \psi, \psi)+\mathcal{O}\left(\|\psi\|^{4}\right) \\
= & -\varepsilon \partial_{t} \psi_{c}-\varepsilon^{2} \partial_{t} \psi_{s}+\varepsilon \Lambda \psi_{c}+\varepsilon^{2} \Lambda \psi_{s}+\varepsilon^{3} T\left(\psi_{c}, \psi_{c}, \psi_{c}\right) \\
& +\varepsilon^{2} B\left(\psi_{c}, \psi_{c}\right)+2 \varepsilon^{3} B\left(\psi_{c}, \psi_{s}\right)+\varepsilon^{4} B\left(\psi_{s}, \psi_{s}\right)+\mathcal{O}\left(\varepsilon^{4}\right) .
\end{aligned}
$$

The construction and estimation of the approximation is made local in Fourier space. To eliminate the $\mathcal{O}\left(\varepsilon^{2}\right)$-terms which are not time derivatives $\left(\partial_{t}=\varepsilon^{2} \partial_{T}\right)$ or which are related to the linear part of the Ginzburg-Landau equation we choose

$$
\Lambda \psi_{s}=-B\left(\psi_{c}, \psi_{c}\right)
$$

Since the Fourier spectrum of $\Lambda$ for the appearing wave numbers lies in the left half plane, this equation for $\psi_{s}$ can be solved in $Q X$. Therefore, we can write $\hat{\Lambda}^{-1}=\hat{\Lambda}^{-1}\left(k, \varepsilon^{2}\right) \in \mathscr{L}\left(Z_{0}, Z_{r_{Z}}\right)$ for $k \in I_{2}=\left[-2 k_{c}-2 \varrho / 3,-2 k_{c}+\right.$ $2 \varrho / 3] \cup[-2 \varrho / 3,+2 \varrho / 3] \cup\left[2 k_{c}-2 \varrho / 3,2 k_{c}+2 \varrho / 3\right]$. To eliminate the term of order $\mathcal{O}\left(\varepsilon^{3}\right)$ for $k$ close to $\pm k_{c}$ we choose the coefficient $\gamma$ in the GinzburgLandau equation such that

$$
\gamma e^{i k_{c} y}=2 p_{\varphi_{1}} B\left(a, \Lambda^{-1} \mathrm{~B}(a, a)\right)+p_{\varphi_{1}} T(a, a, a),
$$


where $a=\hat{\varphi}\left(k_{c}, 0\right) e^{i k_{c} y}+\hat{\varphi}\left(-k_{c}, 0\right) e^{-i k_{c} y}$. Compare the estimate of $s_{3}$ in (10). lemma.

The properties of the approximation $\psi$ are summarized in the following

Lemma 8. Let $A_{1} \in C\left(\left[0, T_{0}\right], H_{l, u}^{3}(\mathbb{C})\right)$ be the solution of the GinzburgLandau equation (7). Then the approximation $\psi=\varepsilon \psi_{c}+\varepsilon^{2} \psi_{s}$ from above satisfies $E_{s} \psi_{c}=0, E_{c} \psi_{s}=0$ and

$$
\begin{aligned}
& \sup _{t \in\left[0, T_{0} / \varepsilon^{2}\right]}\left\|\psi_{0}-\psi\right\|_{X}=\mathcal{O}\left(\varepsilon^{2}\right), \quad \sup _{t \in\left[0, T_{0} / \varepsilon^{2}\right]}\left(\left\|\psi_{s}\right\|_{X^{1}}+\left\|\psi_{c}\right\|_{X^{1}}\right)=\mathcal{O}(1), \\
& \sup _{t \in\left[0, T_{0} / \varepsilon^{2}\right]}\left\|E_{s}(\operatorname{Res}(\psi))\right\|_{X}=\mathcal{O}\left(\varepsilon^{5 / 2}\right), \quad \sup _{t \in\left[0, T_{0} / \varepsilon^{2}\right]}\left\|E_{c}(\operatorname{Res}(\psi))\right\|_{X}=\mathcal{O}\left(\varepsilon^{7 / 2}\right) .
\end{aligned}
$$

Proof. By construction it is clear that $E_{s} \psi_{c}=E_{c} \psi_{s}=0$. Since $\left\|S_{\varepsilon} A_{1}\right\|_{L_{l ; u}^{2}(\mathbb{C})} \leq C\left\|A_{1}\right\|_{H_{l ; u}^{3}(\mathbb{C})}$ and $\varphi_{ \pm 1} \in \mathscr{L}\left(L_{l ; u}^{2}(\mathbb{C}), H_{l ; u}^{m}\left(Z_{r_{Z}}\right)\right)$ for all $m \in \mathbb{N}$ by Lemma 5 we get $\left\|\psi_{c}\right\|_{X^{1}}=\mathcal{O}(1)$. Therefore $\left\|B\left(\psi_{c}, \psi_{c}\right)\right\|_{X}=\mathscr{O}(1)$. Since $\hat{\Lambda}^{-1}\left(k, \varepsilon^{2}\right) \in \mathscr{L}\left(Z_{0}, Z_{r_{Z}}\right)$ for $k \in I_{2}$ can be extended smoothly by zero to a multiplier $\hat{\Lambda}^{-1}$ with $\left\|\left(1+|\cdot|^{2}\right)^{m / 2} \hat{\Lambda}^{-1}\right\|_{C_{b}^{2}\left(\mathbb{R}, \mathscr{L}\left(Z_{0}, Z_{r Z}\right)\right)}<C$ for all $m \in \mathbb{N}$ we also have $\left\|\psi_{s}\right\|_{X^{1}} \leq\left\|\psi_{s}\right\|_{H_{l ;:}^{m}\left(Z_{r_{Z}}\right)}=\mathcal{O}(1)$.

We start now with estimating the terms in $\operatorname{Res}(\psi)$. All terms which are formally of order $\mathcal{O}\left(\varepsilon^{4}\right)$ are of order $\mathcal{O}\left(\varepsilon^{4}\right)$ without expanding anything with respect to $\varepsilon$ and so this is true in the $L_{l ; u}^{2}\left(Z_{0}\right)$-norm, too, because $\left\|\psi_{s}\right\|_{X^{1}}=\left\|\psi_{c}\right\|_{X^{1}}=\mathcal{O}(1)$.

The time derivative $\partial_{t} \psi_{s}$ can be estimated by

$$
\left\|\varepsilon^{2} \partial_{t} \psi_{s}\right\|_{X}=\varepsilon^{4}\left\|\partial_{T} \psi_{s}\right\|_{X}=\left\|2 \varepsilon^{4} \Lambda^{-1} B\left(\psi_{c}, \partial_{T} \psi_{c}\right)\right\|_{X} \leq C \varepsilon^{4}\left\|\psi_{c}\right\|_{Y}\left\|\partial_{T} \psi_{c}\right\|_{Y}
$$

The last factor of this line can be estimated by

$$
\begin{aligned}
\partial_{T} \psi_{c} & =\partial_{T}\left[\varphi_{1}\left(A_{1}(\varepsilon y) e^{i k_{c} y}\right)\right]+\partial_{T}\left[\varphi_{-1}\left(A_{-1}(\varepsilon y) e^{-i k_{c} y}\right)\right] \\
& =\varphi_{1}\left(e^{i k_{c} y} \partial_{T} S_{\varepsilon} A_{1}\right)+\varphi_{-1}\left(e^{-i k_{c} y} \partial_{T} S_{\varepsilon} A_{-1}\right)
\end{aligned}
$$

and $\partial_{T} A_{1}$ by the right hand side of the Ginzburg-Landau equation,

$$
\begin{aligned}
\left\|\partial_{T} S_{\varepsilon} A_{1}\right\|_{L_{l ; u}^{2}} & \leq C\left\|S_{\varepsilon} A_{1}\right\|_{L_{l ; u}^{2}}+C\left\|S_{\varepsilon} \partial_{Y}^{2} A_{1}\right\|_{L_{l ; u}^{2}}+C\left\|S_{\varepsilon} A_{1}\right\|_{H_{l ; u}^{1}}^{3} \\
& \leq C\left\|A_{1}\right\|_{H_{l ; u}^{3}}^{3}+C\left\|A_{1}\right\|_{H_{i ; u}^{3}}^{3} .
\end{aligned}
$$

Summarized we have that $\varepsilon^{2}\left\|\partial_{t} \psi_{s}\right\|_{X}$ is of order $\mathcal{O}\left(\varepsilon^{4}\right)$ is the $X$-norm, too. It remains to estimate the terms which are not of order $\mathcal{O}\left(\varepsilon^{4}\right)$, namely

$$
\operatorname{Res}_{1}=\varepsilon \partial_{t} \psi_{c}+\varepsilon \Lambda \psi_{c}+2 \varepsilon^{3} B\left(\psi_{c}, \psi_{s}\right)+\varepsilon^{3} T\left(\psi_{c}, \psi_{c}, \psi_{c}\right) .
$$

Applying $E_{s}$ on $\operatorname{Res}_{1}$, we get

$$
\left\|E_{s}\left(\operatorname{Res}_{1}\right)\right\|_{X}=\left\|2 \varepsilon^{3} B\left(\psi_{c}, \psi_{s}\right)+\varepsilon^{3} E_{s} T\left(\psi_{c}, \psi_{c}, \psi_{c}\right)\right\|_{X} \leq \mathcal{O}\left(\varepsilon^{5 / 2}\right) .
$$


Because of Lemma 5 and due to the real problem it is sufficient to estimate $\left\|p_{\varphi_{1}}\left(\operatorname{Res}_{1}\right)\right\|_{L_{i ; u}^{2}(\mathbb{C})}$ instead of $\left\|E_{c}\left(\operatorname{Res}_{1}\right)\right\|_{X}$. Defining $\hat{\chi}_{c}(k)=\hat{\chi}_{0}\left(k-k_{c}\right)$, we get finally with $p_{\varphi_{1}}\left(\varphi_{1} S_{\varepsilon} A_{1}\right)=\chi_{c} S_{\varepsilon} A_{1}$

$$
\begin{aligned}
p_{\varphi_{1}}\left(\operatorname{Res}_{1}\right)= & \varepsilon^{3} \chi_{c}\left(\partial_{T} S_{\varepsilon} A_{1} e^{i k_{c} y}\right)+\varepsilon \chi_{c} \lambda_{1}\left(S_{\varepsilon} A_{1} e^{i k_{c} y}\right) \\
& +\varepsilon^{3} 2 p_{\varphi_{1}} B\left(\psi_{c},-\Lambda^{-1} B\left(\psi_{c}, \psi_{c}\right)\right)+\varepsilon^{3} p_{\varphi_{1}} T\left(\psi_{c}, \psi_{c}, \psi_{c}\right) .
\end{aligned}
$$

We subtract the scaled Ginzburg-Landau equation, after applying $\chi_{c}$ on it, from $p_{\varphi_{1}}\left(\operatorname{Res}_{1}\right)$ and get

$$
p_{\varphi_{1}}\left(\operatorname{Res}_{1}\right)=s_{1}+s_{2}+s_{3}
$$

with

$$
\begin{aligned}
s_{1}= & \chi_{c}\left(\left(\varepsilon^{3} \partial_{T} S_{\varepsilon} A_{1}-\varepsilon^{3} \partial_{T} S_{\varepsilon} A_{1}\right) e^{i k_{c} y}\right)=0, \\
s_{2}= & \chi_{2}\left(\varepsilon \lambda_{1}\left(S_{\varepsilon} A_{1} e^{i k_{c} y}\right)-\left[\varepsilon ^ { 3 } \left(\left(\lambda_{0}+i v_{0}\right) S_{\varepsilon} A_{1}\right.\right.\right. \\
& \left.\left.\left.-\left(\lambda_{2}+i v_{2}\right) \varepsilon^{-2} \partial_{y}^{2} S_{\varepsilon} A_{1}\right)\right] e^{i k_{c} y}\right), \\
s_{3}= & \chi_{c}\left(\varepsilon^{3} p_{\varphi_{1}} 2 B\left(\psi_{c},-\Lambda^{-1} B\left(\psi_{c}, \psi_{c}\right)\right)+\varepsilon^{3} p_{\varphi_{1}} T\left(\psi_{c}, \psi_{c}, \psi_{c}\right)\right) \\
& -\varepsilon^{3} \gamma\left|S_{\varepsilon} A_{1}\right|^{2} S_{\varepsilon} A_{1} e^{i k_{c} y} .
\end{aligned}
$$

Note that $\left(1-\chi_{0}\right)^{(n)}(0)=0$ for all $n \in \mathbb{N}$. Therefore by Lemma 7 we have for $A \in H_{l ; u}^{3}$

$$
\begin{aligned}
\left\|\left(1-\chi_{0}\right) S_{\varepsilon} A\right\|_{L_{l ; u}^{2}} & \leq C\left\|\left(1-\chi_{0}(\varepsilon \cdot)\right)\left(1+|\cdot|^{2}\right)^{-3 / 2}\right\|_{C_{b}^{2}}\left\|S_{\varepsilon}\right\|_{\mathscr{L}\left(L_{l, u}^{2}, L_{l, u}^{2}\right.}\|A\|_{H_{l, u}^{3}} \\
& =\mathcal{O}\left(\varepsilon^{5 / 2}\right) .
\end{aligned}
$$

The multiplier for $s_{2}$ is given in Fourier space by

$$
\hat{M}(k, \varepsilon)=\left(\hat{\lambda}_{1}(k)-\left[\varepsilon^{2}\left(\lambda_{0}+i v_{0}\right)+\left(\lambda_{2}+i v_{2}\right)\left(k-k_{c}\right)^{2}\right]\right) \hat{\chi}_{c}(k)
$$

and so $\hat{M}\left(k+k_{c}, \varepsilon^{2}\right)=\mathcal{O}\left(|k|^{3}+\varepsilon^{4}+\varepsilon^{2}|k|\right)$ for $k, \varepsilon \rightarrow 0$. As in Lemma 7 we get $\left\|s_{2}\right\|_{L_{l ; u}^{2}(\mathbb{C})}=\mathscr{O}\left(\varepsilon^{7 / 2}\right)$.

By using several times the triangle inequality and applying Lemma 7 we get $\left\|s_{3}\right\|_{L_{l ; u}^{2}(\mathbb{C})}=\mathcal{O}\left(\varepsilon^{7 / 2}\right)$ uniformly in time on the required time interval. For the whole calculation for $s_{3}$ we refer to [Sch92a]. We need $m=3$ in $A \in H_{l ; u}^{m}$ to get high enough powers of $\varepsilon$ in the estimate for $s_{2}$ and for (11) which is needed for $s_{3}$.

What remains is the comparison of the approximation $\psi_{0}$ used in the theorem and the above approximation $\psi$. Applying Lemma 7 on $\hat{\varphi}\left(k, \varepsilon^{2}\right)-$ $\hat{\varphi}\left(k_{c}, 0\right)$ the required estimate follows. 


\subsection{The estimates for the error}

Now we have all ingredients to show Theorem 2 . We know already that the quadratic terms generate the main difficulties. Since higher order terms are not problematic the following calculations are also valid when not only quadratic terms appear. We can write the quadratic terms of $Q N(u)$ as a symmetric bilinear form $B(u, u)$. Therefore we consider again the system

$$
\partial_{t} u=\Lambda u+B(u, u)
$$

We make now an approximation $\psi$ with the properties of Lemma 8 . We have constructed operators to separate the critical and the uncritical modes of the error made by the approximation $\psi$. The right scaling would be at a time one $\varepsilon^{1 / 2}$-order higher for the critical resp. uncritical modes than the related modes of the approximation $\psi$. Therefore, we suppose the error to be the sum

$$
R(x, t, \varepsilon)=\varepsilon^{3 / 2} R_{c}(x, t, \varepsilon)+\varepsilon^{5 / 2} R_{s}(x, t, \varepsilon)
$$

with $R_{c}=E_{c}^{h} R_{c} \in Q X$ and $R_{s}=E_{s}^{h} R_{s} \in Q X$. By later calculations we show that such an ansatz is possible. We insert now

$$
u=\psi+R=\varepsilon \psi_{c}+\varepsilon^{2} \psi_{s}+\varepsilon^{3 / 2} R_{c}+\varepsilon^{5 / 2} R_{s}
$$

into equation (5). After dividing by $\varepsilon^{3 / 2}$ and after selecting linear and nonlinear terms, and terms for which the application of the operator $E_{c}$ vanishes, we get the equation

$$
\begin{aligned}
\partial_{t} R_{c}+\varepsilon \partial_{t} R_{s}= & \Lambda R_{c}+\varepsilon \Lambda R_{s}+\varepsilon \tilde{L}_{2}\left(R_{c}\right)+\varepsilon^{2} \tilde{L}_{1}(R) \\
& +\varepsilon^{3 / 2} \tilde{N}_{2}\left(R_{c}\right)+\varepsilon^{5 / 2} \tilde{N}_{1}(R)+\frac{1}{\varepsilon^{2}} \operatorname{Res}(\psi),
\end{aligned}
$$

where the abbreviations stand for

$$
\begin{array}{ll}
\tilde{L}_{2}\left(R_{c}\right)=2 B\left(R_{c}, \psi_{c}\right), & \tilde{L}_{1}(R)=2 B\left(R_{c}, \psi_{s}\right)+2 B\left(R_{s}, \psi_{c}\right), \\
\tilde{N}_{2}\left(R_{c}\right)=B\left(R_{c}, R_{c}\right), & \tilde{N}_{1}(R)=B\left(R_{s}, 2 R_{c}+2 \varepsilon^{1 / 2} \psi_{s}+\varepsilon R_{s}\right) .
\end{array}
$$

If all $R_{s}, R_{c}, \psi_{c}$ and $\psi_{s}$ are of order $\mathcal{O}(1)$ in $Y$, then $\tilde{L}_{1}, \tilde{L}_{2}, \tilde{N}_{1}$, and $\tilde{N}_{2}$ are of order $\mathcal{O}(1)$ in $Q X$, too. To get equations for $R_{c}$ and $R_{s}$ we apply the mode-filters $E_{c}$ and $E_{s}$. Looking at the support of the Fourier modes of $\tilde{L}_{2}\left(R_{c}\right)$ and $\tilde{N}_{2}\left(R_{c}\right)$ we have $E_{c} \tilde{L}_{2}\left(R_{c}\right)=E_{c} \tilde{N}_{2}\left(R_{c}\right)=0$. This is due to the following lemma.

Lemma 9. Let $u, v \in(Q X)^{\beta}$. Then $E_{c}^{h} B\left(E_{c}^{h} u, E_{c}^{h} v\right)=0$.

Proof. We know already that $E_{c}^{h} u \in H_{l ; u}^{m}\left(Z_{r_{Z}}\right) \subset Y$ for $m \in \mathbb{N}$ sufficiently large. Therefore we can consider $B=Q N_{2}$ with $N_{2}$ as a symmetric bilinear 
mapping $H_{l ; u}^{m}\left(Z_{r_{Z}}\right) \times H_{l ; u}^{m}\left(Z_{r_{Z}}\right) \rightarrow L_{l ; u}^{2}\left(Z_{0}\right)$ which is local in the $y$-variable. Again we consider $u=l-\sum \tau_{n} u_{n}$ with $u_{n}(y)=u(y-n) \chi(y)$. Since $\hat{Q} \in$ $C_{b}^{2}\left(\mathbb{R}, \mathscr{L}\left(Z_{0}, Z_{0}\right)\right)$ and $\hat{E}_{c}^{h} \in C_{b}^{2}\left(\mathbb{R}, \mathscr{L}\left(Z_{0}, Z_{r_{Z}}\right)\right)$ we have $E_{c}^{h} Q u=l-$ $\sum \tau_{n} E_{c}^{h} Q u_{n}$ by Lemma 5 . Similarly we have

$$
E_{c}^{h} B\left(E_{c}^{h} u, E_{c}^{h} v\right)=l-\sum E_{c}^{h} Q N_{2}\left(\tau_{n} E_{c}^{h} Q u_{n}, \tau_{m} E_{c}^{h} Q v_{m}\right) .
$$

Looking at the Fourier transform of $E_{c}^{h} Q N_{2}\left(\tau_{n} E_{c}^{h} Q u_{n}, \tau_{m} E_{c}^{h} Q v_{m}\right)$, we see that $\mathscr{F} E_{c}^{h} Q u_{n}$ and $\mathscr{F} E_{c}^{h} Q v_{m}$ are functions with support $I_{c}=\left[-k_{c}-\varrho\right.$, $\left.-k_{c}+\varrho\right] \cup\left[k_{c}-\varrho, k_{c}+\varrho\right]$. The Fourier transform of $N_{2}$ gives a convolution of these terms with result in $H^{2}\left(Z_{0}, 0\right)$ and support $I_{2}=\left[-2 k_{c}-2 \varrho\right.$, $\left.-2 k_{c}+2 \varrho\right] \cup[-2 \varrho,+2 \varrho] \cup\left[2 k_{c}-2 \varrho, 2 k_{c}+2 \varrho\right]$. Since $E_{c}^{h}$ and $Q$ act as multipliers the application of $E_{c}^{h}$ yields zero, since $I_{2} \cap I_{c}=\varnothing$.

We separate now (14) in two parts and define $R_{c}$ and $R_{s}$ to be the solutions of the system

$$
\begin{aligned}
& \partial_{t} R_{c}=\Lambda R_{c}+\varepsilon^{2} L_{c}(R)+\varepsilon^{5 / 2} N_{c}(R)+\varepsilon^{2} \delta_{c}, \\
& \partial_{t} R_{s}=\Lambda R_{s}+L_{s}\left(R_{c}\right)+\varepsilon^{1 / 2} N_{s}(R)+\delta_{s}
\end{aligned}
$$

with the abbreviations

$$
\begin{array}{ll}
\delta_{c}=\left(1 / \varepsilon^{7 / 2}\right) E_{c}(\operatorname{Res}(\psi)), & \delta_{s}=\left(1 / \varepsilon^{5 / 2}\right) E_{s}(\operatorname{Res}(\psi)) \\
L_{c}(R)=E_{c}\left(\tilde{L}_{1}(R)\right), & L_{s}\left(R_{c}\right)=E_{s}\left(\tilde{L}_{2}\left(R_{c}\right)\right), \\
N_{c}(R)=E_{c}\left(\tilde{N}_{1}(R)\right), & N_{s}(R)=E_{s}\left(\varepsilon^{1 / 2} \tilde{L}_{1}(R)+\tilde{N}_{2}\left(R_{c}\right)+\varepsilon \tilde{N}_{1}(R)\right)
\end{array}
$$

and the initial data $\left(R_{1}(0), R_{2}(0)\right)=(0,0)$. Adding the two equations of (15) we get (14). We remark that all terms in the equation for $R_{c}$ vanish under the application of $\left(1-E_{c}^{h}\right)$ and the term in the equation for $R_{s}$ under the application of $\left(1-E_{s}^{h}\right)$. We solve this system in the space

$$
\mathscr{B}_{(Q X)^{\beta}}=C\left(\left[0, T_{0} / \varepsilon^{2}\right],(Q X)^{\beta}\right)^{2}
$$

for a $0<\beta<1$ with the norm

$$
\left\|\left(R_{1}, R_{2}\right)\right\|_{\mathscr{B}_{(Q X)^{\beta}}}=\sum_{i=1}^{2} \sup _{0 \leq t \leq T_{0} / \varepsilon^{2}}\left\|R_{i}\right\|_{(Q X)^{\beta} .}
$$

In the same way we define analogous with $X$ and $Q X$ instead of $(Q X)^{\beta}$. We have to show that the solution is of order $\mathcal{O}(1)$ in $\mathscr{B}_{(Q X)^{\beta}}$. We do that by inverting the linear part of (15) and by applying a contraction principle.

Because of Lemma 8 the inhomogeneity $\delta=\left(\delta_{c}, \delta_{s}\right)$ is obviously in $\mathscr{B}_{Q X}$ and of order $\mathcal{O}(1)$. The nonlinearity $N=\left(N_{c}(R), N_{s}(R)\right)$ is a sum of linear and bilinear terms and therefore a local Lipschitz-continuous mapping from $\mathscr{B}_{(Q X)^{\beta}}$ to $\mathscr{B}_{Q X}$ which maps bounded sets of $\mathscr{B}_{(Q X)^{\beta}}$ in bounded sets in $\mathscr{B}_{Q X}$. 
What remains is the estimation of the solutions of the system

$$
\begin{aligned}
& \partial_{t} R_{c}=\Lambda R_{c}+\varepsilon^{2} L_{c}(R)+\varepsilon^{2} f_{c} \\
& \partial_{t} R_{s}=\Lambda R_{s}+L_{s}\left(R_{c}\right)+f_{s}
\end{aligned}
$$

for $f_{c}=E_{c}^{h} f_{c}$ and $f_{s}=E_{s}^{h} f_{s}$ with $f=\left(f_{c}, f_{s}\right) \in \mathscr{B}_{Q X}$. The existence of solutions of this system is clear. By a fixed point argument the existence of solutions of (16) follows on small time intervals. More interesting is the question of the $\mathcal{O}(1)$-boundedness of these solutions on the time interval $\left[0, T_{0} / \varepsilon^{2}\right]$. We will show this by constructing an analytic semigroup in $Q X=Q L_{l ; u}^{2}\left(Z_{0}\right)$ with generator $\Lambda$. To construct the semigroup we have to show a resolvent estimate. The separation in critical and uncritical modes was done to get an exponentially damped part of the semigroup which gets rid of the too low $\varepsilon$-orders. They do not appear by Lemma 9 in the equation for the exponentially increased modes $R_{c}$. We summarize the results in the following lemma.

Lemma 10. There exists a $\sigma_{0}>0$, such that for the semigroup $e^{\Lambda t}$ in $Q X$ generated by $\Lambda$ we have the estimates

$$
\left\|e^{\Lambda t} E_{s}^{h}\right\|_{\mathscr{L}\left(Q X,(Q X)^{\beta}\right)} \leq C \max \left(1, t^{-\beta}\right) e^{-\sigma_{0} t}
$$

and

$$
\left\|e^{\Lambda t} E_{c}^{h}\right\|_{\mathscr{L}\left(Q X,(Q X)^{\beta)}\right.} \leq C e^{C \varepsilon^{2} t} .
$$

Proof. We have to show that $\Lambda$ is a sectorial operator. By assumption (A2) it is a closed operator in $Q X$. By Lemma 5 and assumption (A4) we have

$$
\left\|\left.(s-\Lambda)^{-1}\right|_{\mathscr{L}(Q X, Q X)} \leq C\right\|(s-\hat{\Lambda}(k))^{-1} \|_{C_{b}^{2}\left(\mathbb{R}, \mathscr{L}\left(Z_{0}, Z_{0}\right)\right)} \leq C /|s-a|
$$

for $s$ in the sector $\bigcap_{k} S(k)$. Therefore, $\Lambda$ is a sectorial operator and $\left(e^{\Lambda t}\right)_{t \geq 0}$ is well defined by a Dunford integral (see [He81]).

To get the required estimates we start with the $E_{c}^{h}$-part. Here we use the representation

$$
p_{\varphi_{1}}\left(e^{\wedge t} E_{c}^{h} u\right)(y)=\iint e^{{\hat{\lambda_{1}}}_{1}\left(k, \varepsilon^{2}\right) t} e^{i k(y-s)} \hat{\chi}_{c}(k) d k p_{\varphi_{1}}(u(s)) d s,
$$

where $\hat{\chi}_{c}(k)=\hat{\chi}_{0}\left(k-k_{c}\right)$. Because of $\left\|E_{c}^{h} u\right\|_{(Q X)^{\beta}} \leq C\left\|p_{\varphi_{1}} u\right\|_{\left.L_{l ; u}^{2} \mathbb{C}\right)}$ the result follows from

$$
\int\left|\int e^{\hat{\lambda}_{1}\left(k, \varepsilon^{2}\right) t} e^{i k y} \hat{\chi}_{c}(k) d k\right| d y \leq C e^{C \varepsilon^{2} t}
$$

due to the compact support of $\hat{\chi}_{c}$. 
To show the estimate for the $E_{s}^{h}$-part we notice that $E_{s}^{h}$ is a $\Lambda$-invariant operator. It remains to estimate

$$
\left\|(s-\Lambda)^{-1} E_{s}^{h}\right\|_{\mathscr{L}(Q X, Q X)} \leq C\left\|(s-\hat{\Lambda}(k))^{-1} \hat{E}_{s}^{h}\right\|_{C_{b}^{2}\left(\mathbb{R}, \mathscr{L}\left(Z_{0}, Z_{0}\right)\right)}
$$

by $C /|s+\sigma|$ for $s \in \hat{S}=(\bigcap S(k)) \cup\{\mathfrak{R} \geq \geq-\sigma / 2\}$. For $|s|>s_{0}$ or $|k|>k_{0}$, with $s_{0}, k_{0}$ sufficiently large, this follows from assumption (A4). For the remaining compact set of $s \in \hat{S}$ and $k$ it is sufficient to show that $(s-\hat{\Lambda}(k))^{-1} E_{s}^{h}$ exists. This is true by assumption (A3) and by choosing $0<2 \sigma_{0}<\sigma$ independent of $\varepsilon$ which has to be done because of the definition of $E_{s}^{h}$. Using well known facts about analytic semigroups the rest follows (see [He81]).

Using this lemma we obtain from the second equation in (16)

$$
\left.R_{s}(t)=\int_{0}^{t} e^{\Lambda(t-\tau)}\left[\left(L_{s}\left(R_{c}\right)+f_{s}\right)\right](\tau)\right] d \tau \text {. }
$$

With the abbreviations $S_{i}(s):=\sup _{t \leq s}\left\|R_{i}(t)\right\|_{X}$ we find

$$
\begin{aligned}
S_{s}(t) & \leq\left(\int_{0}^{t} C \max \left(1, \tau^{-\beta}\right) e^{-\sigma \tau} d \tau\right)\left(C S_{c}(t)+\|f\|_{\mathfrak{B}_{X}}\right) \\
& \leq C S_{c}(t)+C\|f\|_{\mathfrak{B}_{X}} .
\end{aligned}
$$

Similarly we can estimate the first equation

$$
\begin{aligned}
S_{c}(t) & \leq \varepsilon^{2} \int_{0}^{t} C e^{C \varepsilon^{2}(t-\tau)}\left(S_{c}(\tau)+S_{s}(\tau)+\|f\|_{\mathfrak{B}_{X}}\right) d \tau \\
& \leq \varepsilon^{2} C \int_{0}^{t} S_{c}(\tau) d \tau+C\|f\|_{\mathfrak{B}_{X} .}
\end{aligned}
$$

With help of Gronwall's inequality we see that

$$
S_{c}(t) \leq C\|f\|_{\mathfrak{B}_{X}} e^{C T_{0}}=\mathcal{O}(1) \quad \text { and } \quad S_{s}(t) \leq C\|f\|_{\mathfrak{B}_{X}}=\mathcal{O}(1) .
$$

Now we define the inverse $J$ of the linear part by $R=J f$, if $R$ is a solution

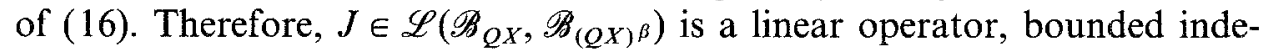
pendently of $\varepsilon$. When we apply $J$ on (15) we obtain

$$
R=\varepsilon^{1 / 2} J N(R)+J \delta=: F(R) .
$$

For $\varepsilon \leq \varepsilon_{0}$ the function $F: \mathscr{B}_{(Q X)^{\beta}} \rightarrow \mathscr{B}_{(Q X)^{\beta}}$ is a contraction on a ball with center $J \delta$ in $\mathscr{B}_{(Q X)^{\beta}}$, because of the Lipschitz-continuity of $N$ and because of the $\varepsilon^{1 / 2}$ in front of $N$. Therefore, there exists a unique fixed point of (18) which is a solution of order $\mathcal{O}(1)$ of (15). By (12) and (13) we have 
constructed a solution of the original problem (14). The estimate of the theorem follows since

$$
\begin{aligned}
\left\|u-\psi_{0}\right\|_{\mathscr{B}_{X}} & \leq\|u-\psi\|_{\mathscr{B}_{Q X}}+\left\|\psi_{0}-\psi\right\|_{\mathscr{P}_{X}} \\
& =\left\|\left(\varepsilon^{3 / 2} R_{c}, \varepsilon^{5 / 2} R_{s}\right)\right\|_{\mathscr{O}_{(Q X)} \beta}+\mathcal{O}\left(\varepsilon^{2}\right) \\
& =\mathcal{O}\left(\varepsilon^{3 / 2}\right) .
\end{aligned}
$$

\section{Application to Bénard's problem}

We put now Bénard's problem (1) in the abstract set-up of Section 2. We remind that Bénard's problem is given by

$$
\begin{array}{ll}
\begin{array}{l}
\partial_{t} u=L_{0} u+L_{1} u+G p+N(u) \\
0=D u=v_{y}+w_{z}
\end{array} & \text { for }(t, y, z) \in[0, \infty) \times \mathbb{R} \times(0, \pi), \\
v_{2}=w=\theta=0 & \text { for }(y, z) \in \mathbb{R} \times\{0, \pi\} .
\end{array}
$$

To apply the above theory we have to write (1) as a dynamical system. Like explained in the introduction the pressure term $G p$ will be eliminated by choosing a space $Q X$, such that the last equation of (1) is automatically fulfilled and the pressure term drops out. To do this we have to construct the projection $Q$ with help of the pressure term $G p$ into the space of divergence free vector fields.

Therefore, we set the linear differential operator $L\left(\varepsilon^{2}\right)=L_{0}+$ $L_{1}\left(\varrho_{c}+\varepsilon^{2}\right)$. It is a continuous mapping from $X^{1}=L_{l ; u}^{2}\left(Z_{2}\right) \cap H_{l ; u}^{1}\left(Z_{1}\right) \cap$ $H_{l ; u}^{2}\left(Z_{0}\right)$ into $X=L_{l ; u}^{2}\left(Z_{0}\right)$, where $Z_{0}=L^{2}\left((0, \pi), \mathbb{R}^{3}\right), Z_{1}=H^{1}\left((0, \pi), \mathbb{R}^{3}\right)$ and $Z_{2}=H^{2}\left((0, \pi), \mathbb{R}^{3}\right) \cap\{$ boundary conditions $\}$. Note that

$$
X=L_{l ; u}^{2}\left(\mathbb{R} \times(0, \pi), \mathbb{R}^{3}\right)
$$

and

$$
X^{1}=H_{l ; u}^{2}\left(\mathbb{R} \times(0, \pi), \mathbb{R}^{3}\right) \cap\{\text { boundary conditions }\} .
$$

It depends smoothly on $\varepsilon^{2}=\varrho-\varrho_{c}$, as $L_{1}$ depends linearly on $\varrho$.

The nonlinear part

$$
N(u)=\left(-v v_{y}-w v_{z},-v w_{y}-w w_{z},-v \theta_{y}-w \theta_{z}\right)
$$

is a bounded polynomial from $Y=H_{l: u}^{1}\left(\mathbb{R} \times(0, \pi), \mathbb{R}^{3}\right) \cap L^{\infty}\left(\mathbb{R} \times(0, \pi), \mathbb{R}^{3}\right)$ to $X$. Therefore, $N \in C^{4}(Y, X)$ and $X^{1} \subset Y \subset X$, because of a generalized version of Sobolev's imbeddings theorems, which follows again by localization. To define the projection $Q$ we state:

(A1) There exists a family of continuous projections $\hat{Q} \in C_{b}^{2}\left(\mathbb{R}, \mathscr{L}\left(Z_{0}, Z_{0}\right)\right)$, such that $Q u=\mathscr{F}^{-1} \hat{Q} \mathscr{F} u=(v, w, \theta)$ fulfills the conditions $\partial_{y} v+\partial_{z} w=0$, 
$[v](y)=\int v(y, z) d z=0$ for all $y \in \mathbb{R},\left.w\right|_{z=0, \pi}=0$ and $Q\left(\partial_{y} p, \partial_{z} p, 0\right)=0$ for all $\left(\partial_{y} p, \partial_{z} p, 0\right) \in L_{l ; u}^{2}\left(Z_{0}\right)$.

Proof. The $\theta$ variable is independent of this projection and it applies only to the hydrodynamic part coming from Navier-Stokes equation. We define this part of $Q$ by the solutions $u=(v, w)$ of the system $u+\nabla p=f$; $\nabla \cdot u=0$ under the conditions $\left.w\right|_{z=0, \pi}=0$ and $[v](y)=0$ for all $y \in \mathbb{R}$. We first eliminate for fixed $y$ the mean value $\left[f_{1}\right](y)=\int f_{1}(y, z) d z$ of the first component $f_{1}$ of $f$. This can always be done by a $z$-independent pressure $p_{0}(y)$. We have $p_{0}^{\prime}(y)=\left[f_{1}\right](y)$. This is equivalent to subtract the mean value in the Fourier-transformed problem of the term $f_{1}$. Now, the system $u+\nabla p=\tilde{f}, \nabla \cdot u=0$ with $[v]=\left[\tilde{f}_{1}\right]=[p]=0,\left.w\right|_{0, \pi}=0$ has to be solved. To define $\hat{Q}$ we study the Fourier-transformed system,

$$
v+i k p=\tilde{f}_{1}, \quad w+\partial_{z} p=\tilde{f}_{2}, \quad i k v+\partial_{z} w=0,
$$

which can be solved by an expansion $v=\sum_{m=1}^{\infty} v_{m} \cos m z, \quad w=$ $\sum_{m=1}^{\infty} w_{m} \sin m z, \tilde{f}_{2}=\sum_{m=1}^{\infty}\left(f_{2}\right)_{m} \sin m z, \tilde{f}_{1}=\sum_{m=1}^{\infty}\left(f_{1}\right)_{m} \cos m z$ and $p=$ $\sum_{m=1}^{\infty} p_{m} \cos m z$. Since $[v]=\left[\tilde{f}_{1}\right]=0$ the corresponding terms with $m=0$ drop out. By inserting this expansion we get three-dimensional invariant systems $v_{m}+i k p_{m}=f_{1 m}, w_{m}-m p_{m}=f_{2 m}, i k v_{m}+m w_{m}=0$. The solution reads

$$
\left(\begin{array}{c}
v_{m} \\
w_{m}
\end{array}\right)=A_{m}(k)\left(\begin{array}{l}
f_{m} \\
g_{m}
\end{array}\right)=\frac{1}{m^{2}+k^{2}}\left(\begin{array}{cc}
m^{2} & -i m k \\
-i m k & k^{2}
\end{array}\right)\left(\begin{array}{l}
f_{m} \\
g_{m}
\end{array}\right) .
$$

The matrices $A_{m}(k)$ and its derivatives with respect to $k$ can be estimated uniformly in $m$ and in $k$ and hence $\hat{Q} \in C_{b}^{2}\left(\mathbb{R}, \mathscr{L}\left(Z_{0}, Z_{0}\right)\right)$.

Therefore, we find

$$
\begin{gathered}
Q X=\left\{(v, w, \theta) \in L_{l ; u}^{2}\left((0, \pi) \times \mathbb{R}, \mathbb{R}^{3}\right) \mid v_{y}+w_{z}=0,\right. \\
\left.\left.w\right|_{z=0, \pi}=0,[v](y)=0 \quad \forall y \in \mathbb{R}\right\}
\end{gathered}
$$

and

$$
\begin{aligned}
(Q X)^{1}= & \left\{(v, w, \theta) \in H_{l ; u}^{2}\left((0, \pi) \times \mathbb{R}, \mathbb{R}^{3}\right) \mid v_{y}+w_{z}=0,\right. \\
& \left.\left.w\right|_{z=0, \pi}=0,[v](y)=0 \forall y \in \mathbb{R}\right\} \cap\{\text { boundary conditions }\} .
\end{aligned}
$$

(A2) To show that $\Lambda$ is a closed operator in $Q X$ with domain of definition $D(\Lambda)=(Q X)^{1}$ it is sufficient to show that

$$
\left\|(\Lambda-s)^{-1}\right\|_{\mathscr{L}\left(X, X^{1}\right)} \leq C
$$


for some $s \in \mathbb{R}$. If we have shown this, one can imbed $(Q X)^{\beta}$ in $W_{l ; u}^{1, q}(\mathbb{R} \times$ $\left.(0, \pi), \mathbb{R}^{3}\right)$ for $1 / 2<\beta<1$ and $1 / q>(5-4 \beta) / 6$ and in $L^{\infty}\left(\mathbb{R} \times(0, \pi), \mathbb{R}^{3}\right)$ for $3 / 4<\beta<1$. This follows similar to the proof of Th.1.6.1 in [He81]. And so we have $(Q X)^{\beta} \subset Y$ for $\beta>3 / 4$.

Before showing the rest of (A2) we look at

(A3) This assumption is already shown in the introduction.

For general problems the validity of this assumption can only be shown numerically (see also [CH82], chapter 14).

(A4) + (A2) It remains to show (19) and (A4) (a) -(c). The assumption (A4) (a) is stated in

Lemma 11. There exists an $a \in \mathbb{R}$, such that we have for $s \in S(k)=$ $\left\{s \in \mathbb{C} \mid \arg \left(s+a+\left(C_{1} / 2\right) k^{2}\right) \in(-5 \pi / 4,5 \pi / 4)\right\}$ the estimate

$$
\left\|(s-\hat{\Lambda}(k))^{-1}\right\|_{\mathscr{L}\left(Z_{0}, Z_{0}\right)} \leq \frac{C}{\left|C_{1} k^{2}+s+a+1\right|} .
$$

Proof. First we examine the operator $\hat{\Lambda}_{0}=\hat{Q} \hat{L}_{0}$. This means we have to look at the two separated problems

$$
\begin{aligned}
& v_{z z}-k^{2} v-s v+i k p=f \\
& w_{z z}-k^{2} w-s w+\partial_{z} p=g \\
& i k v+w_{z}=0
\end{aligned}
$$

and

$$
\kappa \theta_{z z}-\kappa k^{2} \theta-s \theta=h
$$

under the boundary conditions $\left.v_{z}\right|_{z=0, \pi}=\left.w\right|_{z=0, \pi}=\left.\theta\right|_{z=0, \pi}=0$ and $[v(k)]=$ $0,[f(k)]=0$ for $f, g, h \in L^{2}((0, \pi))$. We treat only the more complicated first problem. Substituting $l=s+k^{2}$, applying $\int(\bar{v}, \bar{w})^{T} \cdots d z$ to the first two equations and integration by parts gives

$$
\int\left(\left|v_{z}\right|^{2}+\left|w_{z}\right|^{2}+l|v|^{2}+l|w|^{2}\right) d z=-\int(f \bar{v}+g \bar{w}) d z .
$$

By looking separately at the real and at the imaginary part the estimate follows for the operator $\hat{\Lambda}_{0}=\hat{Q} \hat{L}_{0}$. Because of

$$
\begin{aligned}
(\hat{\Lambda}-l)=\left(\hat{\Lambda}_{0}+\hat{\Lambda}_{1}-l\right) & =\left(\hat{\Lambda}_{0}-l\right)\left(I d .+\left(\hat{\Lambda}_{0}-l\right)^{-1} \hat{\Lambda}_{1}\right) \\
& =\left(I d .+\hat{\Lambda}_{1}\left(\hat{\Lambda}_{0}-l\right)^{-1}\right)\left(\hat{\Lambda}_{0}-l\right)
\end{aligned}
$$


the operator $\hat{\Lambda}_{1}=\hat{Q} \hat{L}_{1} \in C_{b}^{2}\left(\mathbb{R}, \mathscr{L}\left(Z_{0}, Z_{0}\right)\right)$ is a perturbation of $\hat{\Lambda}_{0}$ for $l$ sufficiently large with help of Neumann series. Since there remains only a compact set of $s$ and $k$ the assertion follows.

Using Lemma 5 it is sufficient to prove $(s-\hat{\Lambda})^{-1}\left(1+|k|^{2}\right) \epsilon$ $C_{b}^{2}\left(\mathbb{R}, \mathscr{L}\left(Z_{0}, Z_{0}\right)\right),(s-\hat{\Lambda})^{-1}\left(1+|k|^{2}\right)^{1 / 2} \in C_{b}^{2}\left(\mathbb{R}, \mathscr{L}\left(Z_{0}, Z_{1}\right)\right)$ and $(s-\hat{\Lambda})^{-1} \in$ $C_{b}^{2}\left(\mathbb{R}, \mathscr{L}\left(Z_{0}, Z_{2}\right)\right)$ for showing (19). To derive this we have to estimate

$$
\begin{aligned}
& \sup _{k \in \mathbb{R}, s \in S(k)}\left\|(s-\hat{\Lambda})^{-1}\left(1+|k|^{2}\right)\right\|_{\mathscr{L}\left(Z_{0}, Z_{0}\right)}, \\
& \sup _{k \in \mathbb{R}, s \in S(k)}\left\|(s-\hat{\Lambda})^{-1}\left(1+|k|^{2}\right)^{1 / 2}\right\|_{\mathscr{L}\left(Z_{0}, Z_{1}\right),} \\
& \sup _{k \in \mathbb{R}, s \in S(k)}\left\|(s-\hat{\Lambda})^{-1}\right\|_{\mathscr{L}\left(Z_{0}, Z_{2}\right),} \\
& \sup _{k \in \mathbb{R}, s \in S(k)}\left\|\hat{\Lambda}^{\prime}(s-\hat{\Lambda})^{-1}\right\|_{\mathscr{L}\left(Z_{0}, Z_{0}\right),} \\
& \sup _{k \in \mathbb{R}, s \in S(k)}\left\|\hat{\Lambda}^{\prime \prime}(s-\hat{\Lambda})^{-1}\right\|_{\mathscr{L}\left(Z_{0}, Z_{0}\right),}
\end{aligned}
$$

where we have written $s \in S(k)$ to include also (A4) (b) -(c). Introducing the norm

$$
\|\cdot\|_{X(k)}=|k|^{2}\|\cdot\|_{z_{0}}+|k|\|\cdot\|_{z_{1}}+\|\cdot\|_{z_{2}}
$$

we see that $\sup _{k \in \mathbb{R}}\left\|\hat{\Lambda}^{\prime}(k)\right\|_{\mathscr{L}\left(X(k), Z_{0}\right)}+\sup _{k \in \mathbb{R}} \mid \hat{\Lambda}^{\prime \prime}(k) \|_{\mathscr{L}\left(X(k), Z_{0}\right)} \leq C$. Therefore, (22) follows from

$$
\sup _{s \in S(k), k \in \mathbb{R}}\left\|(s-\hat{\Lambda}(k))^{-1}\right\|_{\mathscr{L}\left(Z_{0}, X(k)\right)} \leq C .
$$

Using (21) it is sufficient to show (23) for $\Lambda_{0}$ instead of $\Lambda$. To make life easy we treat only the more complicated part of (20) and use the fact that this problem possesses a simple expansion of eigenfunctions $v=\sum_{m=1}^{\infty} v_{m} \cos m z, \quad w=\sum_{m=1}^{\infty} w_{m} \sin m z, \quad g=\sum_{m=1}^{\infty} g_{m} \sin m z, \quad f=$ $\sum_{m=1}^{\infty} f_{m} \cos m z \quad$ and $p=\sum_{m=1}^{\infty} p_{m} \cos m z$. With $l=k^{2} / 2+s \epsilon$ $\{l \in \mathbb{C} \mid \arg (l) \in(-5 \pi / 4,5 \pi / 4)\}$ we have to solve

$$
\begin{aligned}
& \left(-m^{2}-k^{2} / 2-l\right) v_{m}+i k p_{m}=f_{m}, \quad\left(-m^{2}-k^{2} / 2-l\right) w_{m}-m p_{m}=g_{m}, \\
& i k v_{m}+m w_{m}=0 .
\end{aligned}
$$

The solutions are given by

$$
\left(\begin{array}{l}
v_{m} \\
w_{m}
\end{array}\right)=A_{m}(k)\left(\begin{array}{l}
f_{m} \\
g_{m}
\end{array}\right)=\frac{-1}{\left(m^{2}+k^{2}\right)\left(m^{2}+k^{2} / 2+l\right)}\left(\begin{array}{cc}
m^{2} & -i m k \\
-i m k & k^{2}
\end{array}\right)\left(\begin{array}{l}
f_{m} \\
g_{m}
\end{array}\right)
$$

and so the assertion follows. Note the constant $C_{1} / 2$ in the definition of $S(k)$ in contrast to $C_{1}$ in the resolvent estimate (A4) (a). It is used here. 
We end this paper with a remark about the approximation of the pressure.

Remark 12. It is possible to approximate the pressure $p$ by the pressure related to the eigenfunction $\tilde{\varphi}\left(k_{c}\right) e^{i k_{c} y}$. Doing that we get formally

$$
p=\varepsilon p_{\psi_{0}}+\mathcal{O}\left(\varepsilon^{2}\right)=\frac{3}{2} \varepsilon \cos z e^{i k_{c} y} A_{1}(\varepsilon y)+c . c .+\mathcal{O}\left(\varepsilon^{2}\right) .
$$

Since $p_{0}^{\prime}$ in the proof of (A1) depends only on the nonlinearity it is of order $\mathcal{O}\left(\varepsilon^{2}\right)$. But clearly $p_{0}$ itself is not integrable and so it makes only sense to approximate the pressure gradient in this problem. Inserting $u=\varepsilon \psi+$ $\varepsilon^{3 / 2} R$ of the proof of Theorem 2 in $\nabla p=\partial_{t} u-L u-N(u)$ we see that $\nabla p$ can be approximated by $\nabla p_{\psi_{0}}$ up to an error $\mathcal{O}\left(\varepsilon^{3 / 2}\right)$ in the norm of $L_{l ; i}^{2}\left(\mathbb{R} \times(0, \pi), \mathbb{R}^{3}\right)$.

\section{Acknowledgements}

The author is grateful for discussions with A. Mielke and A. van Harten. The research was partially supported by Deutsche Forschungsgemeinschaft (DFG) under grant $\mathrm{Ki} 131 / 5-1$ and by the European Community under SC1-CT91-0670.

\section{References}

[CH82] S.-N. Chow and J. K. Hale, Methods of Bifurcation Theory. Springer, Berlin 1982.

[CE90] P. Collet and J.-P. Eckmann, The time dependent amplitude equation for the Swift-Hohenberg problem. Comm. Math. Phys. 132, 139-153 (1990).

[diPES71] R. C. di Prima, W. Eckhaus and L. A. Segal, Nonlinear wave-number interaction in near-critical two-dimensional flows. J. Fluid Mech. 49, 705-744 (1971).

[Eck92] W. Eckhaus, The Ginzburg -Landau equation is an attractor. Preprint 746, University Utrecht (1992).

[He81] D. Henry, Geometric theory of semilinear parabolic equations. Lect. Notes in Maths 840, Springer, Berlin 1981.

[IMD89] G. Iooss, A. Mielke and Y. Demay, Theory of steady Ginzburg-Landau equation, in hydrodynamic stability problems. Eur. J. Mech., B/Fluids, 8, nr. 3, 229-268 (1989).

[KSM92] P. Kirrmann, G. Schneider and A. Mielke, The validity of modulation equations for extended systems with cubic nonlinearities. Proc. Royal Soc. Edinburgh 122A, 85-91 (1992).

[Mi92] A. Mielke, Reduction of PDE's on domains with several unbounded directions. J. Appl. Math. Phys. (ZAMP) 43 449-470 (1992).

[NW69] A. Newell and J. Whitehead, Finite bandwidth, finite amplitude convection. J. Fluid Mech. 38 279-303 (1969).

[Sch92a] G. Schneider, Die Gültigkeit der Ginzburg-Landau-Approximation. Dissertation, Universität Stuttgart 1992.

[Sch92b] G. Schneider, A new estimate for the Ginzburg-Landau approximation on the real axis. J. Nonlin. Sci., to appear 1994.

[VI92] A. Vanderbauwhede and G. Iooss, Center-manifold in infinite dimensions. Dynamics Reported 1, 125-162. Springer-Verlag, Berlin 1992.

[vH91] A. van Harten, On the validity of Ginzburg-Landau's equation. J. Nonlin. Sci. 1, 397-422 (1991).

[vH92] A. van Harten, Decay to the Ginzburg - Landau manifold. Lecture held in Stuttgart, July 1992. 


\section{Abstract}

Modulation equations play an essential role in the understanding of complicated dynamical systems near the threshold of instability. Here we look at systems defined over domains with one unbounded direction and show that the Ginzburg-Landau equation dominates the dynamics of the full problem, locally, at least over a long time-scale. As an application of our approximation theorem we look here at Bénard's problem. The method we use involves a careful handling of critical modes in the Fourier-transformed problem and an estimate of Gronwall's type.

(Received: February 26, 1993; revised: July 17, 1993) 